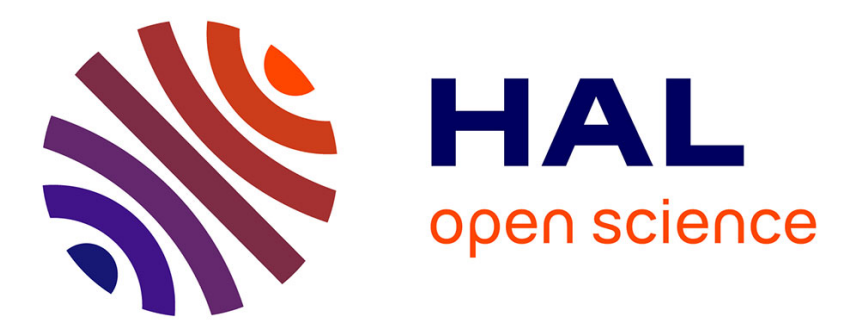

\title{
La place des valeurs dans le développement professionnel d'étudiants de Master : point de vue des stagiaires et de leurs référents.
}

\author{
Dominique Broussal, Mathieu Chabridon
}

\section{- To cite this version: \\ Dominique Broussal, Mathieu Chabridon. La place des valeurs dans le développement professionnel d'étudiants de Master: point de vue des stagiaires et de leurs référents.. Travail et apprentissages: revue de didactique professionnelle, 2009, 3, pp.26-43. hal-00762071}

\section{HAL Id: hal-00762071 \\ https://hal.science/hal-00762071}

Submitted on 6 Dec 2012

HAL is a multi-disciplinary open access archive for the deposit and dissemination of scientific research documents, whether they are published or not. The documents may come from teaching and research institutions in France or abroad, or from public or private research centers.
L'archive ouverte pluridisciplinaire HAL, est destinée au dépôt et à la diffusion de documents scientifiques de niveau recherche, publiés ou non, émanant des établissements d'enseignement et de recherche français ou étrangers, des laboratoires publics ou privés. 
Broussal, D., Chabridon, M. (2009a). La place des valeurs dans le développement professionnel d'étudiants de Master : point de vue des stagiaires et de leurs référents. Travail et apprentissages, 3, 2643.

\title{
La place des valeurs dans le développement professionnel d'étudiants de Master : point de vue des stagiaires et de leurs référents
}

\author{
Dominique BROUSSAL, \\ Mathieu CHABRIDON \\ CREFI-T, Evacap ${ }^{1}$ \\ Université de Toulouse \\ UTM, F-31000, France
}

\section{Résumé}

La situation d'alternance vécue par les étudiants d'un Master Professionnel, pris pour objet d'étude, est au cœur de dynamiques identitaires participant à leur développement professionnel. Nous avons cherché dans une perspective exploratoire à montrer en quoi les conflits de valeurs induits par cette situation participaient, au-delà de la dimension identitaire, au développement escompté. Un double regard sur ce questionnement est proposé dans cet article. Celui des référents professionnels tout d'abord, qui ont été interrogés sur le positionnement professionnel des stagiaires. Celui des étudiants ensuite, à travers l'analyse d'écrits rédigés à l'issue des séances de clinique ayant lieu au cours de la formation. Les divergences dégagées sont articulées en conclusion et permettent de proposer un certain nombre de perspectives théoriques ou méthodologiques.

Mots clés : expérience axiologique, développement professionnel, formation, valeurs, conflits, professionnalité

\section{Abstract}

The situation of work-based learning lived by the students of a professional Master, taken as object of our research, is the center of identity constructs which take part in their professional development. We tried to show in an exploratory prospect, how the values conflicts sparked off by the situations participated in the expected development, beyond the dimension of identity. A double view is proposed in this article. First of all we shall be

\footnotetext{
${ }^{1}$ Centre de Recherches en Education, Formation, Insertion de Toulouse, EVAluation et Professionnalisation des Acteurs et des Contextes (CREFI-T/EVACAP) dirigé par Anne Jorro, Professeure, France.
} 
interested in the professional referents who were questioned about the professional location of the trainees. In the second part we shall be interested in the student's point of view, by analyzing papers written after clinical sessions during the training. The clear differences are questioned in conclusion and it allows to propose theoretical or methodological perspectives.

Keywords : axiological experience, professional development, training, values, professionality

\section{Introduction}

Les deux études qui suivent explorent la place tenue par les valeurs dans le parcours d'étudiants suivant un Master «Conseil en développement professionnel » à l'Université de Toulouse II. L'investigation privilégie l'expérience vécue lors du stage en entreprise. Celui-ci comprend 320 heures, réparties sur une durée de quatre mois. Les deux études ont été menées en parallèle, selon des méthodologies et des perspectives sensiblement différentes. La première recherche est centrée sur le point de vue des professionnels qui accompagnent les étudiants sur leur terrain de stage : elle décrit la façon dont ces référents apprécient le développement attendu au regard des positionnements professionnels perçus, et s'appuie sur une série de dix entretiens. La seconde recherche présente le point de vue des étudiants eux-mêmes. A défaut d'ouvrir sur une analyse comparative, la mise en relation de ces différents regards nous conduit à préciser en conclusion un certain nombre de questions théoriques et méthodologiques que pose notre objet d'étude. Nous nous proposons d'esquisser de façon introductive les contours de cet objet dans les lignes qui suivent, avant d'y revenir plus précisément dans la présentation des recherches.

L'objectif de professionnalisation revendiqué par le Master «Conseil en développement professionnel » place les étudiants qui s'y engagent dans une situation d'alternance, au cœur de laquelle se succèdent ou s'entrecroisent des logiques, des contraintes et des préoccupations qui spécifient chacun des deux univers en contact : celui du travail et celui de la formation. Parmi les nombreuses recherches qui portent sur l'un ou l'autre de ces champs, nous nous inscrivons dans le prolongement des travaux qui se sont intéressés aux dynamiques identitaires dans des contextes de tensions ou de conflits, dans des périodes de crise ou de reconfiguration parfois intenses (Sainsaulieu, 1988 ; Dubar, 1994, 2006 ; Legault, 2003 ; Bourgeois, 2006 ; Kaddouri, Lespessailles, Maillebouis, Vasconcellos, 2008 ; Osty, 2008). Dans son ouvrage fondateur pour les recherches sur l'identité au travail, Sainsaulieu (1988) souligne que «l'expérience des relations est si intense et durable dans les organisations contemporaines que leurs effets culturels ne se limitent pas aux seules normes de comportement dans les bureaux et les ateliers » (p. 13) : le travail occupe ainsi un rôle de premier plan dans la formation des identités. Sainsaulieu évoque par ailleurs le rôle déterminant joué par les conflits relationnels dans la découverte de soi en société. S'intéressant à la socialisation professionnelle, Dubar (2006) décrit de son côté la dynamique de déstructuration/restructuration qui sous-tend la «reconversion périodique des identités » (p. 239), prenant parfois l'allure d'une crise des identités. Il met en évidence l'importance des trajectoires individuelles, la dimension biographique de cette dynamique, position qui rompt avec une logique par trop déterministe. Legault (2003) appréhende le processus identitaire à partir de trois composantes : l'identité revendiquée, 
l'identité déclarée et l'identité reconnue. Analysant les conflits d'identité qui peuvent survenir, il indique que «dans certaines circonstances, nous devrons faire des choix difficiles entre des valeurs personnelles (résultant de l'appropriation de l'appartenance familiale) et des valeurs institutionnelles ou organisationnelles » (op. cit., p. 24). Dans une publication récente, Osty (2008) réaffirme la centralité du travail dans la construction des identités et pose que "l'activité de travail s'effectue dans un cadre normatif où l'accès à la reconnaissance et l'intégration sociale sont conditionnés par la conformité à des modèles culturels valorisés par l'entreprise » (p. 81). S'intéressant enfin à l'engagement en formation, Bourgeois (2006) étudie quant à lui l'hypothèse selon laquelle les tensions identitaires pourraient «devenir source d'engagement effectif et de motivation dans le processus de formation»(p. 66). Dans l'ensemble de ces recherches la question de l'articulation des valeurs individuelles aux normes collectives est posée. Est aussi pointée la dimension centrale des conflits de valeurs dans les processus de « déstructuration/restructuration » des identités.

Le déplacement que nous avons choisi d'opérer dans notre projet de recherche, consiste à envisager ces conflits non pas au regard du rôle qu'ils jouent dans les dynamiques identitaires, mais en interrogeant le lien qu'ils peuvent entretenir avec le développement professionnel des individus, celui-ci étant entendu comme un ensemble de transformations intervenant à «l'intérieur des dispositifs de professionnalisation » et revêtant un caractère opératoire, cognitif, affectif et identitaire (Wittorski, 2007, p. 105). Un tel déplacement infléchit considérablement l'objet de recherche. En effet si la «reconversion périodique des identités », pour reprendre les termes de Dubar, n'est pas un but affirmé des dispositifs de professionnalisation, le développement professionnel des individus constitue quant à lui un de leurs objectifs prioritaires, objectif que s'approprient les formés eux-mêmes. En quoi les valeurs et conflits de valeurs participent-ils donc du développement escompté ? C'est la question que les deux études suivantes se proposent d'explorer.

\section{Point de vue des référents sur le positionnement professionnel des stagiaires}

La valeur professionnalisante des stages est reconnue par les référents professionnels (Bedin, 2009). Cette première étude décrit le regard que ceux-ci portent sur les conflits de valeurs vécus par les étudiants accueillis. Ces conflits résultent notamment de la nécessité pour les stagiaires d'avoir à acquérir des valeurs légitimes, partagées et participant à la structuration de l'activité dans ces organisations : les valeurs professionnelles. Pour conduire notre étude, nous nous sommes entretenus avec dix référents sur le positionnement professionnel des stagiaires au regard de ces valeurs professionnelles. Nous pensons en effet que le positionnement des stagiaires résulte en partie des transformations liées aux conflits de valeurs éprouvés sur les lieux de stage. Le point de vue des référents professionnels sur ce positionnement apparaît dès lors primordial, si l'on veut comprendre l'enjeu que recouvre l'acquisition par les stagiaires de ces valeurs, dans une perspective de développement professionnel.

\subsection{La question des valeurs professionnelles dans la formation Master}

La formation dispensée aux étudiants vise leur développement professionnel à travers une double acquisition : 
- $\quad$ celle de compétences professionnelles. Ces dernières sont d'ailleurs bien identifiées et formalisées à travers cinq grandes catégories de compétences : compétences en ingénierie de formation, compétences théoriques, méthodologiques, de communication et enfin compétences éthiques.

celle d'une "culture professionnelle". Il s'agit de l'ensemble des connaissances et des valeurs professionnelles nécessaires à l'exercice d'une profession. Si les connaissances à acquérir sont bien identifiées dans le cadre de ce Master professionnel, ce n'est par contre pas le cas des valeurs.

Plusieurs raisons peuvent être avancées quant à la difficulté d'identifier les valeurs professionnelles du conseil. Tout d'abord, la profession du conseil (dans l'acception libérale et savante de la notion de profession) n'existe pas à proprement parler. Il existerait plutôt une pluralité d'activités de conseil, peut-être autant que de lieux d'exercice de cette activité... Ainsi, plus que les valeurs d'une profession, les valeurs professionnelles dont il est question ici seraient d'abord les valeurs des organisations dans lesquelles les étudiants font leur stage. Mais là aussi, il s'agit d'être prudent. On sait en effet l'instrumentalisation qui peut être faite du concept anthropologique de culture (Cuche, 2004), par des organisations affichant certaines valeurs à des fins utilitaristes de management et de communication. Ainsi, nous définirons les valeurs professionnelles comme un ensemble de valeurs légitimes et partagées dans une organisation, et participant à l'activité professionnelle au sein de celle-ci.

Malgré cette difficulté, il ne fait aucun doute que l'acquisition de ces valeurs professionnelles est un des objectifs de cette formation en alternance. Une acquisition qui ne doit pas simplement permettre aux étudiants de s'adapter à une organisation, mais bien de se transformer en vue d'une insertion professionnelle. Ainsi, nous avançons que c'est en agissant sur leur lieu de stage que les étudiants font l'expérience de ces valeurs, en se confrontant à elles plus ou moins directement. Cette expérience axiologique, traversée par de nombreux conflits, participe alors à la structuration du positionnement professionnel des stagiaires.

\subsection{L'expérience axiologique des étudiants en stage génératrice d'un positionnement professionnel}

La nécessité d'acquérir des valeurs professionnelles oblige les stagiaires à certaines réorganisations subjectives. Celles-ci s'accompagnent de conflits de valeurs parfois importants qui les sollicitent de l'intérieur. Nous présenterons pour commencer quelques éléments explicatifs sur la construction de ces conflits dans une perspective sociologique. Puis nous montrerons ensuite que c'est l'extériorisation de ces conflits et des transformations conjointes dans l'agir des stagiaires, que le positionnement professionnel indique.

\section{- L'émergence des conflits de valeurs dans la rencontre du stagiaire et du contexte professionnel}

L'émergence des conflits de valeurs opérerait donc dans l'expérience des stagiaires. Nous proposons de penser celle-ci comme la rencontre d'un individu construit socialement et du contexte professionnel dans lequel il agit.

L'individu est ici appréhendé à travers ses habitudes sociales. Celles-ci englobent l'ensemble des valeurs, des goûts et des postures incorporés lors de sa socialisation 
primaire notamment. Nous nous rapprochons ce faisant de deux concepts élaborés par Bourdieu (1980). Celui d'« habitus » d'abord, recouvrant un ensemble de dispositions d'un groupe d'agents qui donnent une même signification à leur pratique, mais aussi un système de définition du monde. Celui d'« hexis corporelle » en deuxième lieu, désignant un ensemble de dispositions pratiques corporelles comme les manières de se tenir, de parler et de marcher. Cet ensemble de valeurs incorporées par la socialisation et s'extériorisant en situation jouerait ainsi un rôle significatif dans l'activité des individus, le plus souvent de façon non conscientisée. Boudon (1999) développe un point de vue différent sur la question : en ce qui le concerne, les valeurs participent aussi à l'action, mais à travers ce qu'il nomme une «rationalité axiologique ». Les valeurs fonctionneraient ainsi comme une véritable théorie pour les individus, théorie à laquelle ils se réfèreraient quant à leurs choix et leurs décisions pour orienter leur action. Bien que ces deux auteurs ne se rejoignent pas quant à leur explication théorique, ils pointent tous les deux les liens étroits qu'entretiennent les valeurs et l'action, rejoints en cela par de nombreux travaux.

Le contexte professionnel est quant à lui appréhendé à travers certaines valeurs constituant un système de référence à l'action. Ces valeurs participent ainsi à la structuration de l'organisation et de l'activité professionnelle : ce sont les valeurs affichées avec des visées de communication et de management, ce sont aussi des valeurs plus spécifiques liées à un métier et/ou une profession. Elles participent alors à guider, orienter voire justifier l'action des professionnels, s'apparentant ainsi au genre (Clot, 2004) de l'activité professionnelle. Ce sont ces valeurs multiples, hétérogènes et parfois contradictoires au sein d'un même contexte professionnel, mais toutefois légitimes, reconnues et partagées par les différents acteurs, que nous nommons valeurs professionnelles.

La rencontre entre le stagiaire et le contexte professionnel dans lequel il agit, désire s'intégrer et se développer professionnellement, est génératrice de conflits de valeurs qui ne prennent jamais réellement fin. Ces conflits naissent notamment de l'hétérogénéité, voire de la contradiction entre plusieurs "types" de valeurs en présence : valeurs individuelles nées de la socialisation, valeurs de la profession et/ou de l'organisation, valeurs projetées sur une profession et/ou un métier et ne résistant pas en situation. Les approches sociologiques de la construction des identités offrent des éclairages précieux à ce sujet. Nous retenons ici les travaux de Dubar mentionnés plus haut (2006), montrant comment les conflits de valeurs participent aux processus identitaires biographiques (dialogue conflictuel entre identité héritée et identité projetée) et relationnels (dans lequel les valeurs participent aux transactions identitaires des acteurs et à leur reconnaissance). Nous reconnaissons par ailleurs l'importance des affects dans la transformation de soi (Barbier \& Galatanu, 1998) ainsi que celle de la corporéité dans l'agir des individus (Joas, 1997). Ces deux composantes semblent toutefois faiblement prises en compte dans la logique d'action sous-tendue par les processus identitaires précédemment cités, alors qu'elles interviennent selon nous dans le positionnement des stagiaires.

\section{- Le positionnement professionnel des stagiaires}

En agissant au sein d'un contexte professionnel, l'individu va éprouver mais aussi déployer certaines valeurs, professionnelles ou non. Ainsi, agir au sein d'un contexte reviendrait toujours à se positionner vis-à-vis de celui-ci. L'expérience créée par cet agir, que nous qualifions d'axiologique, permet aux stagiaires d'éprouver des conflits de valeurs qui peuvent être un frein important au développement professionnel. En effet, face à ces conflits engendrant souvent de la souffrance (Lahire, 2001), certains individus peuvent se retirer, fuir, ou adopter une posture de repli, voire de protection. D'autres arrivent à "faire 
avec", à "faire face" à ces conflits et prennent alors le risque désiré de changer, de se transformer. Une telle transformation ne peut s'effectuer sans ré-élaboration de sens. Ce sont ainsi de nouvelles valeurs qui s'élaborent au regard des valeurs déjà-là. Elles viennent modifier le cadre des futures actions des stagiaires, indiquant le chemin à parcourir en balisant un espace dans lequel ils vont pouvoir et/ou devoir agir. Cette expérience axiologique participe alors du changement du stagiaire et ainsi de la construction de son positionnement professionnel.

\subsection{Méthodologie : une recherche exploratoire}

Notre travail théorique nous permet d'affirmer l'intérêt heuristique d'une recherche qui vise à éclairer la façon dont les référents professionnels reconnaissent le positionnement professionnel des stagiaires. Toutefois, les données recueillies à ce stade de la recherche ne permettent pas encore de mettre à l'épreuve des hypothèses de recherche. C'est donc dans une perspective exploratoire que nous avons envisagé notre méthodologie. De façon à recueillir des données qualitatives, nous avons construit une grille d'entretien semi-directif à partir de l'opérationalisation des notions de positionnement et de valeurs professionnelles.

$\mathrm{Au}$ cours des mois de février à avril 2008, nous nous sommes entretenus avec 10 référents professionnels : 5 hommes et 5 femmes. La prise de contact s'est effectuée par mail ou par téléphone. Ces référents sont issus d'organisations de différentes natures : associations, entreprises, institutions privées et publiques. Les fonctions exercées à l'intérieur de ces organisations sont elles aussi diverses : assistant de recrutement, directeur, responsable, formateur, chef de projet, conseiller technique supérieur, etc. Avant chaque entretien, nous avons rappelé qu'il ne s'agissait en aucun cas d'une évaluation déguisée des stagiaires accueillis, et encore moins d'une évaluation des lieux de stage. Toutefois, nous pouvons raisonnablement supposer qu'il est difficile pour des référents d'écarter cette dimension évaluative.

Les entretiens visaient à faire expliciter par les référents professionnels le rôle et la place des valeurs professionnelles dans leur organisation et, au regard de celles-ci, leurs attentes vis-à-vis du stagiaire. Il s'agissait aussi de savoir comment les référents appréhendaient le désir du stagiaire à se professionnaliser ainsi que sa possibilité et/ou capacité à identifier et mobiliser les valeurs professionnelles. Les entretiens ont duré une vingtaine de minutes en moyenne. Enregistrés, ils ont été ensuite retranscrits en intégralité et ont fait l'objet d'une analyse thématique (Bardin, 2003) de façon à dégager les premiers éléments d'une trame d'analyse des positionnements des stagiaires par les référents professionnels.

\subsection{Résultats}

D'une façon générale, la mobilisation d'une part des valeurs promues par l'organisation (dans les discours, les écrits...), et d'autre part des valeurs comme attitudes significatives pour les référents (prise d'initiative, manière de parler...), paraît rendre compte pour les référents du positionnement professionnel des étudiants. De façon à illustrer nos propos, nous faisons le choix de citer de larges extraits des entretiens, qui contiennent certains items retenus dans le cadre de l'analyse thématique mais sans nous y limiter, et ce afin que le lecteur puisse lui-même porter un regard analytique sur ceux-ci. 


\section{- Un rapport normatif au positionnement des stagiaires}

La mobilisation des valeurs promues par l'organisation, qui sont plus ou moins affichées selon les lieux dans lesquels ils effectuent leur stage, renseigneraient les référents sur le positionnement professionnel des stagiaires, dans le sens d'une inscription de l'individu dans un espace professionnel balisé par certaines valeurs structurantes. Dans ce sens, un des référents exprime la nécessité de s'approprier ces valeurs, de pouvoir s'en revendiquer de façon à ne pas se situer en dehors de la structure, en dehors du «cadre » :

«n'importe quel membre de l'association s'approprie ces valeurs parce que sinon elle est forcément, elle se situerait en dehors quelque part de la structure s'il n'y avait pas ces idées de valeurs, si elle n'était pas capable de s'en revendiquer, en tout cas d'intégrer et de respecter les valeurs de l'entreprise, de la structure. Pour moi c'est un impératif mais c'est aussi quelque chose qui doit être là naturellement, qu'on n'a pas besoin de forcer » (Référent G).

Certains référents font part de leurs attentes vis-à-vis de cette mobilisation dans les dires des stagiaires et le mémoire professionnel :

«Si ... dans leur mémoire, ou dans leur rapport, puisqu'ils me soumettent au fur et à mesure leur travail ... Si jamais effectivement il n'y a de référence à ces valeurs, je m'en étonne un peu. Je leur dis, attendez, il faudrait peut-être que, justement, votre étude, votre diagnostic, vos préconisations, votre problématique, s'inscrivent dans cette culture qui est la nôtre » (Référent $\mathrm{C}$ ).

Bien que celles-ci puissent être mobilisées de façon plus ou moins rhétorique (Tardif et Lessard, 1999) et/ou stratégique (De Certeau, 1981), la déclaration des valeurs rendrait compte d'un positionnement professionnel, voire d'une potentialité à se développer professionnellement dans l'organisation lorsqu'elles sont mobilisées dans les premiers entretiens ouvrant le stage. La plupart des référents font ainsi allusion aux entretiens préalables au stage ou réalisés en tout début, dans lesquels eux-mêmes exprimeraient certaines valeurs professionnelles et analyseraient en retour dans la parole du stagiaire des éléments de son positionnement vis-à-vis de celles-ci :

«Il y a vraiment déjà dans les entretiens qui se font au préalable, on se met quand même d'accord sur qui on est véritablement, sur ce qu'on représente, que les choses soient très claires entre le stagiaire et nous déjà en amont. Quand je prends un stagiaire il y a toujours une phase d'explication sur qui nous sommes, nos missions, comment nous nous positionnons, qu'est-ce qu'on véhicule comme valeurs » (Référent $\mathrm{H})$.

\section{- Un rapport compréhensif au positionnement des stagiaires}

Les «valeurs agies » (Jorro, 1999) par les étudiants, qui se déploieraient dans certaines attitudes significatives pour les référents, permettraient à ces derniers d'identifier un positionnement professionnel en construction. Ces «valeurs agies» semblent parfois osciller entre valeurs personnelles et valeurs professionnelles. Elles rendraient ainsi compte de l'investissement subjectif du stagiaire dans l'organisation et pourraient faire davantage l'objet d'une "forme intersubjective de reconnaissance professionnelle" (Bedin, 2009), qui passe notamment par la valorisation du stagiaire. A titre d'illustration, le référent $\mathrm{H}$ explique :

«De la même façon ... vous la voyez à l'accueil là. Voilà les valeurs. Parce que bon, on est une structure associative, ... on est parfois un petit peu en réduction d'effectifs. Ça c'est une valeur professionnelle de solidarité par exemple. Elle est 
stagiaire. ... Mais elle est arrivée elle se met au standard. L'assistante administrative est en congé, on est en réduction d'effectifs, on n'a même pas eu besoin de lui demander. Ca c'est une vraie valeur ça, ça c'est important, ça c'est important».

C'est aussi le Référent E qui semble identifier certaines valeurs dans des manières de dire ou des manières de faire du stagiaire accueilli :

«Elle va réagir en disant ben oui ça, enfin c'est plus dans le partage, une sorte de, voilà en disant cette manière de dire, cette manière de présenter les choses, je trouve ça bien. Elle donne son avis, on échange là-dessus quoi. Je ne sais plus ce sur quoi c'était l'autre fois. Elle réagissait comme ça»

Si dans les cas précédents, certaines attitudes identifiées par les référents rendraient compte du déploiement par les stagiaires de certaines valeurs professionnelles, ces attitudes renseigneraient le plus souvent sur l'envie, le désir du stagiaire de se professionnaliser. Ainsi, c'est la valeur que le stagiaire accorde à l'activité professionnelle ou à l'organisation, qui se dégagerait de la qualité du travail, de certaines postures, de l'investissement dans des tâches et/ou de certaines réactions :

"Il n’y a pas forcément des indicateurs précis, il y a des postures, il y a des comportements, on le voit plus comme ça. ... Ce sont des choses toutes simples, c'est l'aisance à intégrer une équipe, à discuter, à se positionner pendant les moments où on est en entreprise, voilà, manger avec les collègues »(Référent $\mathrm{H}$ ).

«Mais moi je vois l'intérêt ou l'envie qu'elle a c'est déjà de s'investir dans cette tâche, l'intérêt qu'elle porte à ce travail quoi. ... Puis dans la façon de la réaliser je dirais avec, avec envie quoi » (Référent E).

Ce rapport plus compréhensif au positionnement des stagiaires laisse entrevoir une centration davantage sur le stagiaire que sur le système de référence à l'action de l'organisation de la part des référents. Les conflits de valeurs pourraient alors potentiellement être reconnus comme participant à la construction du positionnement professionnel du stagiaire et in fine, à son développement professionnel. Le contrôle par les référents du positionnement professionnel du stagiaire, dans un rapport normatif à celui-ci, apparaît ainsi comme une étape nécessaire d'une évaluation de ce même positionnement, qui en permettrait une reconnaissance plus large.

\subsection{Perspectives}

Nous ne sommes pas en mesure de faire ici le lien entre les statuts des référents, le type d'organisation dans laquelle ils travaillent et l'éventualité d'une prédominance d'un discours plutôt normatif ou compréhensif vis-à-vis du positionnement des stagiaires. Pourtant, nous savons que ces caractéristiques sont significatives des formes de reconnaissance professionnelle mobilisées par les référents (Bedin, op. cit.). C'est en tout cas dans l'articulation des rapports dégagés que semblent se dessiner les premiers éléments d'une trame d'analyse des positionnements des stagiaires par les référents professionnels.

Toutefois, notre attention se porte sur le fait que les référents professionnels interrogés signalent qu'il existerait des valeurs, d'ordre plutôt personnel, permettant le développement professionnel dans l'organisation, même en tant que stagiaire. C'est ce que semblerait aussi indiquer, dans d'autres recherches, la place décisive de l'adéquation des valeurs de l'organisation et de la personnalité, contrairement au profil technique affiché, dans le processus de recrutement (Lecointe, 2007). Ainsi, la frontière semble parfois mince 
entre les indicateurs d'un positionnement professionnel dégagés par les référents professionnels, et ceux d'un positionnement plus personnel, qui peut apparaître comme un véritable pré-requis à l'exercice de la profession d'après certains référents, et qui relèvent alors plutôt de la personnalité :

«pour les stagiaires que nous recevons, je dirais qu'a priori ils ont déjà en eux ces valeurs, quelque soit le niveau des valeurs mais, ils sont déjà porteurs de ça sinon tout simplement moi je n'aurais pas envie de m'embêter à les voir » (Référent A).

Dés lors, le fait de dégager certains critères et indicateurs de la composante axiologique du développement professionnel permettrait de « poser les limites de l'acte évaluatif » car « les risques de glissement entre la personne et le professionnel guettent toute pratique évaluative » (Jorro, 2007, p.18).

\section{Conflits de valeurs et développement professionnel : le point de vue des étudiants}

Après avoir envisagé dans la partie qui précède le point de vue des référents, intéressons-nous à présent à celui des stagiaires. Quel regard les étudiants de Master Professionnel portent-ils sur les conflits de valeurs auxquels les périodes de stage les confrontent, et comment les articulent-ils à la perspective de leur propre développement professionnel? Pour explorer cette question, nous avons procédé à l'analyse thématique de 21 écrits d'étudiants. Ces écrits ont été rédigés à l'issue des séances de clinique des pratiques que nous animons dans le cadre de la formation de Master, nous inscrivant dans une conception proche de celle posée par Ardoino (1989) : « est donc proprement clinique aujourd'hui, ce qui veut appréhender le sujet (individuel et/ou collectif) à travers un système de relations (constitué en dispositif, c'est-à-dire au sein duquel le praticien, ou le chercheur, comme leurs partenaires, se reconnaissent effectivement impliqués), qu'il s'agisse de viser l'évolution, le développement, la transformation d'un tel sujet ou la production de connaissances, en soi, comme pour lui ou pour nous » (p. 64). Lors de ces séances de clinique, un étudiant évoque une situation professionnelle préalablement choisie. Le groupe travaille à partir de cet exposé en formulant des questions d'exploration puis en construisant un certain nombre de pistes interprétatives ouvrant sur des enjeux de régulation de l'activité à venir. La consigne donnée pour la rédaction des écrits consiste à choisir une des situations évoquées lors du cycle, puis à produire un commentaire qui rende compte à la fois du travail d'interprétation du groupe et du positionnement professionnel de l'étudiant au regard des enjeux de la situation.

Avant de présenter les résultats de l'analyse thématique, faisons trois remarques préliminaires qui apportent des compléments sur la façon dont cette deuxième recherche appréhende son objet. Nous évoquerons successivement : le choix des situations, la question de l'ethos et du professionnalisme, la mobilisation par les étudiants de cadres théoriques issus de la formation pour penser leur propre développement.

\section{- Des situations d'incertitude, des dilemmes partagés}

Les situations reprises dans les écrits de la clinique sont toutes des situations dans lesquelles s'est posée, pour un acteur singulier, la question du bien fondé de sa conduite : «elle [l'exposante] se retourne vers nous et nous pose une question éthique : jusqu'où peut-on aller dans la demande du client? (5)» rapporte ainsi un étudiant. Les 
interrogations ne remettent pas en cause la compétence du stagiaire, ni l'efficacité de sa décision, mais elles interrogent les systèmes de valeurs qui sous-tendent l'évaluation de son agir. Le dilemme prend la forme d'un conflit de valeurs que les écrits expriment de façons diverses : « conflit cognitif $\left(3^{2}\right) »$, « conflit interne (3)», « il arrive que les deux éthiques entrent en conflit (5)», «des valeurs qui n'étaient pas forcément les miennes, mais véhiculées par mon secteur d'activité et auxquelles on doit à un moment se conformer de façon à ne pas vivre un conflit cognitif trop perturbateur (7) ». Bien que liés à des contextes professionnels spécifiques, les témoignages font largement écho à l'expérience des autres membres. Ils sont l'occasion de discuter collectivement d'une professionnalité de conseiller que le groupe entend partager. A ce titre, la formalisation des valeurs est inséparable d'un enjeu «d'ostension de soi » (Barbier, 2003, p. 139), et participe de l'élaboration des ethos.

\section{- Professionnalisme et ethos}

Comme le rappelle Barbier, la définition que propose Bateson (1971) de l'ethos est celle d'un «système culturellement normalisé d'organisation des instincts et des émotions des individus à l'intérieur d'une culture, et [traduisant] l'affect de cette culture » (cité par Barbier, op. cit., p. 129). Dès lors que les professions se sont constituées, le professionnalisme s'est trouvé étroitement lié au développement de l'ethos, ce «milieu de vie qu'est la pratique professionnelle » (Legault, 1999, p. 6). La lutte pour la reconnaissance sociale imposant aux groupes professionnels d'harmoniser leurs exigences, leurs manières d'agir, leurs valeurs, des communautés de penser et de faire se sont progressivement constituées, leur cohésion se trouvant renforcée par la vitalité des échanges au sein d'une vie collective généralement fournie. Appartenir à une profession c'est ainsi partager un ensemble de valeurs, au premier rang desquelles le professionnalisme, «valeur par excellence de toute profession » (Legault, op. cit., p. 48). Se faire admettre impose dans ces conditions un effort d'acculturation qui peut parfois prendre l'allure d'une conversion (Davis, 1968).

Au gré de la diversification des activités professionnelles, de la dissolution des collectifs, mais aussi sous l'effet conjoint de l'accroissement de la concurrence et de la précarité, la consistance de ces ethos professionnels s'est progressivement délitée, au profit de cultures d'organisations (Aubert \& Gaulejac, 1991, Wittorski, 2007). Or il semble que ces questions d'ethos et de professionnalisme reprennent aujourd'hui de l'importance, au moment où nombre d'observateurs remettent en cause des pratiques de management ou d'organisation du travail que la performance économique suffisait à justifier jusqu'ici. Un des enjeux de la professionnalisation des acteurs et des contextes s'inscrit dans ce contexte : la fonction critique émerge comme réponse professionnelle aux contradictions, voire aux dilemmes que produit la double exigence de compétence et d'obligation de résultats (Jorro, 2005).

Il est intéressant de revenir à l'objet qui nous occupe en gardant présent à l'esprit les éléments ci-dessus. Un certain nombre de caractéristiques propres aux métiers du Conseil se détachent dès lors:

- les pratiques du Conseil présentent une grande hétérogénéité (Lhotellier, 2001) : en s'engageant dans cette voie, les étudiants ont le sentiment d'œuvrer à la reconnaissance d'une profession, bien plus que celui d'entrer dans une communauté professionnelle à l'ethos constitué.

2 Ces indications chiffrées renvoient à l'écrit de clinique d'où la citation est extraite. 
- les stages en entreprises les conduisent vers des champs d'activité extrêmement divers (missions locales, agences d'intérim, services d'animation socioculturelle, etc.). A la différence des stages hospitaliers pour les étudiants en médecine, ou des stages en cabinet pour les futurs avocats, l'étudiant en Conseil en développement professionnel dispose rarement d'un modèle d'identification unique pour construire son ethos professionnel.

- la formation que les étudiants du Master reçoivent à l'Université comporte des enseignements consacrés à l'élaboration des identités sociales et professionnelles, à l'éthique, au développement professionnel. Ils disposent donc pour réfléchir leur propre professionnalisation et pour analyser les changements dans lesquels ils sont engagés, d'un appareil théorique important dont nous verrons qu'ils font usage. La situation nous paraît être assez originale pour mériter d'être soulignée, nous y revenons dans le paragraphe qui suit.

\section{- La théorie comme ressource pour réfléchir sa professionnalisation}

Nous l'évoquions à l'instant, une des originalités du Master professionnel «Conseil en développement professionnel » est de mettre à disposition des étudiants un cadre théorique susceptible de les aider à penser leur propre développement. Les écrits de la clinique témoignent de la mobilisation de ces références, que ce soit à travers une activité définitionnelle : «l'approche de l'éthique professionnelle repose à la fois sur la dimension morale (on ne peut agir sans ignorer les principes et les lois) et sur la dimension éthique (quelle décision est la meilleure dans une situation donnée) (5)», ou bien par la mention explicite de concepts issus des théories présentées en cours : «la responsabilité de l'action au sens de Ricœur, c'est-à-dire considérer que l'on est en mesure de le faire et donc d'assumer la décision prise (11) », «j'y vois là l'idée développée par Richard Wittorski du « processus identitaire » qui permet à l'individu, au collectif, de construire, à travers des expériences et des savoirs, une identité professionnelle (15)», «est-ce cela la solidarité organique dont parle Dubar? (20)».

\subsection{Le positionnement axiologique des stagiaires}

Nous présentons à présent les résultats de l'analyse thématique conduite sur les 21 écrits qui nous ont été communiqués en mai 2008. Les tableaux ci-dessous présentent les catégories qui ont pu être dégagées concernant dans un premier temps l'appréciation de la situation évoquée (tableau 1) puis dans un deuxième temps les catégories de positionnement axiologique référées à la perspective de développement professionnel des acteurs (tableau 2). 
Tableau 1 : appréciation de la situation

\begin{tabular}{|c|c|c|c|}
\hline CATÉGORIES & COMPOSANTES & EXEMPLES & $\begin{array}{c}\text { Nbre } \\
\text { d'items }\end{array}$ \\
\hline \multirow{2}{*}{$\begin{array}{l}\text { RESTRICTION DE } \\
\text { LIBERTE }\end{array}$} & Fragilité & « simple stagiaire », « jeune diplômé » & \\
\hline & $\begin{array}{l}\text { Risque } \\
\text { Soumission } \\
\text { Stress }\end{array}$ & $\begin{array}{l}\text { « revendiquer son éthique }[. . .] \text { cela peut être fatal » } \\
\text { «bon gré, mal gré » } \\
\text { «le stress peut précipiter nos actions » }\end{array}$ & 7 \\
\hline \multirow[t]{4}{*}{$\begin{array}{l}\text { ETHIQUE } \\
\text { CONTEXTUELLE }\end{array}$} & $\begin{array}{l}\text { Circonstances } \\
\text { atténuantes }\end{array}$ & « la dure loi du marché concurrentiel et du travail » & \\
\hline & Réalisme & $\begin{array}{l}\text { « notre idéal étant irréel et non applicable à l'action } \\
\text { du terrain » }\end{array}$ & \\
\hline & $\begin{array}{l}\text { Ethos } \\
\text { Etrangeté }\end{array}$ & $\begin{array}{l}\text { «spécificité de son milieu professionnel » } \\
\text { «le milieu professionnel étant plus étranger pour } \\
\text { nous » }\end{array}$ & 8 \\
\hline & $\begin{array}{l}\text { Action / } \\
\text { environnement }\end{array}$ & « interrogée au regard du contexte et des actes » & \\
\hline $\begin{array}{l}\text { ETHIQUE } \\
\text { FONCTIONNELLE }\end{array}$ & $\begin{array}{l}\text { Rationalité } \\
\text { axiologique }\end{array}$ & $\begin{array}{l}\text { «des techniques de ventes qui font partie de } \\
\text { l'éthique professionnelle » }\end{array}$ & 1 \\
\hline $\begin{array}{l}\text { RUPTURE } \\
\text { AXIOLOGIQUE }\end{array}$ & Incongruité & $\begin{array}{l}\text { « ce qu'elle est en train de vivre est différent, voir } \\
\text { contraire à ce qu'elle apprend lors de sa formation } \\
{[\ldots] \text { ce que l'on nous a inculqué depuis notre }} \\
\text { enfance » }\end{array}$ & 2 \\
\hline
\end{tabular}

Tableau 2 : positionnement axiologique et développement professionnel

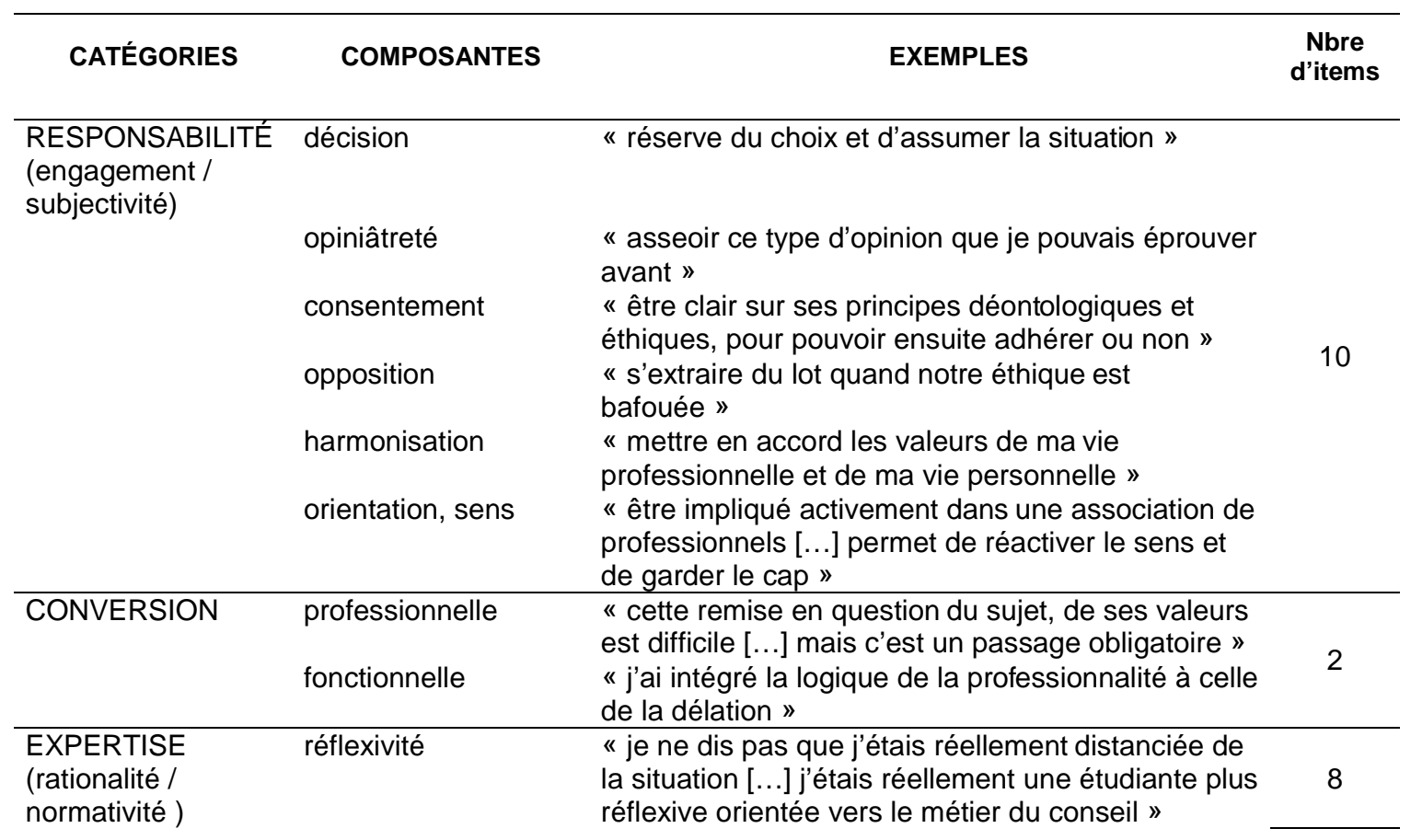


vigilance

interprétation

régulation

partition "le risque de dérive justifie une vigilance vis-à-vis des écarts entre le dire et le faire "

" on retravaille sur les clés d'interprétation »

« mettre des limites aux demandes des clients afin

de respecter la loi »

« mettre une limite entre l'éthique personnelle et

l'éthique du métier »

\subsection{Le choix des figures axiologiques de professionnalité}

Interrogeons-nous à partir de ces résultats sur le statut que les stagiaires accordent à leur propre expérience axiologique. L'analyse thématique permet de soutenir l'hypothèse selon laquelle les conflits de valeurs éprouvés au cours des stages ne seraient pas seulement reconnus comme des étapes obligées de la socialisation professionnelle, mais interviendraient positivement dans la construction de la professionnalité, celle-ci étant entendue comme "capacité à mettre en œuvre une expertise complexe encadrée par un système de références normatives sinon axiologiques » (Braem \& Aballéa, 2002, p. 8). Les propos des étudiants montrent que ces conflits vécus sur le terrain de stage, ou partagés dans le groupe de clinique, sont l'occasion d'une réorganisation des préférences, d'une clarification des systèmes de valeurs, et d'une mise en dialogue de ces systèmes de valeurs avec un ethos professionnel en construction. Ce processus de positionnement axiologique dont les écrits rendent compte, est à la fois réflexif et prospectif. Il nous paraît opérer à deux niveaux qu'il importera de confirmer et de préciser dans des recherches ultérieures :

- celui des hiérarchies axiologiques : l'expérience des tensions que le stagiaire éprouve en s'engageant dans une activité dont il est « redevable » (Jorro, 2007, p. 25) et dans laquelle sa perspective professionnelle le projette, suscite un travail de réorganisation des valeurs entre elles. Ainsi, un comportement qui dans le système de valeurs hérité des formes de socialisation antérieures était associé à de la délation, pourra par exemple, à l'issue du repositionnement des préférences, être référé à des normes organisationnelles (l'intérêt commun) et valorisé au nom de l'efficacité du service.

- le deuxième niveau est celui de la mise en regard de ce travail individuel de hiérarchisation avec le façonnement des identités collectives auquel il contribue. Ce processus est éminemment évaluatif, les valeurs intervenant ici en tant que « rapport entre les rapports » (Barbier, 2003, p. 124). Il ne s'agit pas à ce niveau d'opposer les valeurs entre elles (préférer le sens de l'honneur à l'intérêt du service, la rentabilité à la générosité), mais de faire émerger les métavaleurs (Ferry, 2002, p. 51) qui subsument les délibérations locales. De cette élection subjective de métavaleurs dépendent les modalités selon lesquelles l'individu règlera, au cours du processus de « déconstruction/reconstruction », les rapports entre valeurs personnelles et normes collectives, identités privées et identités professionnelles. Ce processus nous semble d'autant plus difficile à documenter et à décrire qu'il s'entremêle aux diverses dynamiques de professionnalisation (reconnaissance, affiliation, insertion, apprentissage, socialisation) dans lesquelles le stagiaire en formation est pris. Nos premières analyses nous conduisent à envisager que ce processus labile d'élection se concrétise progressivement à travers le choix d'une figure axiologique de professionnalité. Ce choix opère parmi un ensemble de figures, qui se différencient tout d'abord par la plus ou moins grande importance qu'elles font aux valeurs individuelles dans l'évaluation et la régulation de l'agir. Elles se distinguent ensuite en 
fonction de la possibilité admise ou refusée de faire entendre ces valeurs individuelles dans l'évolution des normativités qui encadrent l'activité de travail. Dans la perspective que nous esquissons, le conflit de valeurs n'est pas tant envisagé sous l'angle de perturbations identitaires à dépasser, que sous celui d'un agir professionnel engageant la subjectivité de l'acteur : le «choix difficile» entre valeurs personnelles et valeurs institutionnelles (Legault, 2003) que nous évoquions plus haut, relève dans une telle conception d'un positionnement individuel au regard de la responsabilité estimée, du pouvoir d'agir perçu, de la subjectivité assumée.

L'élaboration théorique qui précède postule donc une nature bi-dimensionnelle de l'expérience axiologique des stagiaires. Les conflits de valeurs sont partie prenante du développement professionnel en ce qu'ils accompagnent le travail de réorganisation des systèmes de valeurs par la prise en compte progressive des contraintes spécifiques aux milieux de travail. Cette réorganisation conduit à une intégration plus ou moins aboutie des normes régissant les pratiques. Une deuxième modalité d'ancrage des valeurs au développement professionnel émerge : c'est celle qui opère à travers le choix d'une figure axiologique de professionnalité. Nous n'excluons pas que ces deux niveaux entrent euxmêmes en conflit, le choix de la figure de professionnalité constituant en toute fin l'instance régulatrice. Ces éléments de questionnement une fois posés, revenons avant de conclure à la description de ces deux figures axiologiques de professionnalité que nous proposons.

\subsection{Le professionnel responsable, le professionnel expert}

La dynamique de développement initiée par la situation de conflit fait émerger, nous l'évoquions, deux principales figures axiologiques de professionnalité. La troisième figure, celle de la conversion, est peu représentée dans le groupe étudié. Ces deux figures organisent le rapport entre systèmes de valeurs et agir, elles sont emblématiques d'une conception de la professionnalité en laquelle les étudiants se projettent et se reconnaissent. Nous les avons désignées comme figure $d u$ professionnel responsable et figure $d u$ professionnel expert. Quelles conceptions ces deux figures recouvrent-elles dans les écrits des étudiants?

\section{- Le professionnel responsable}

La figure que nous qualifions de professionnel responsable est celle qui affirme la contribution des conflits de valeurs à la vie professionnelle. La fonction critique est ici reconnue comme élément de compétence. La conception de la responsabilité que cette figure axiologique sous-tend est celle d'un engagement de l'acteur dans sa globalité, impliquant à la fois ethos professionnel, normes appropriées et valeurs héritées. Rejoignant pleinement la définition de Durand (1999) selon laquelle il y a dilemme «lorsque se présentent des conflits de principes, des conflits de valeurs et où, même après réflexion, aucun principe ni aucune valeur s'impose », (cité par Massé, 2003, p. 53), le professionnel responsable assume la part subjective de sa décision. La professionnalité ne tient par pour lui du gommage, de la neutralisation ou de la conversion des identités privées, mais au contraire de l'acceptation du caractère opératoire de cette conflictualité des identités, des valeurs et des normes que le travail impose et dont il s'enrichit. Cette figure rejoint la nécessité exprimée par certains cadres d'entreprise de «mettre en accord des engagements dans certains projets de l'entreprise avec des valeurs et des héritages culturels et personnels qui comptent »(Courpasson \& Dany, 2008). Un tel exercice de la responsabilité est considéré comme produisant un bénéfice partagé : en limitant l'autonomisation possible de 
l'action, l'emprise des contexte, il garantit d'une certaine façon le sens des activités de travail, permettant pour les acteurs de "garder le cap », ainsi que l'indique une étudiante. En reprenant les différentes dimensions du développement professionnel relevées par Wittorski (op. cit.) et évoquées plus haut, opératoires, cognitives, affectives et identitaires, nous remarquerons que l'identification de cette figure du professionnel responsable conduit à questionner de façon inédite les aspects affectifs et identitaires du développement professionnel, en remettant en cause l'a priori positif du changement. La préservation des valeurs héritées, la relative permanence de son identité privée peuvent en effet apparaître comme de réels enjeux professionnels pour l'étudiant en cours de professionnalisation, et non plus comme des obstacles.

\section{- Le professionnel expert}

A l'origine de cette deuxième figure se trouve une préoccupation récurrente que nous paraît clairement pointer Cifali (1996) : « Une question se pose constamment dans l'agir : "Est-ce bien, est-ce mal ?". Elle est même première chez les débutants, avec l'espoir d'un jugement qui pourrait départager et surtout protéger du mal »(p. 124). C'est à un tel « espoir » de jugement que répond selon nous la figure du professionnel expert : face aux conflits de valeurs qui surviennent, il s'agit pour le professionnel expert de reconnaître le primat de la normativité et de la rationalité en ce qui concerne la conduite, l'évaluation et la régulation des activités de travail. Contrairement à la situation précédente, les dimensions identitaires et affectives sont alors pleinement engagées dans le processus de développement, et les valeurs héritées ainsi que les résistances identitaires sont considérées comme des obstacles, au sens quasi bachelardien du terme (1938). La résolution experte des conflits anticipe projectivement sur une dé-personnalisation réussie, une mise en suspens de la subjectivité au profit de la réflexivité et de la rationalité constituées en compétence.

\section{Conclusion}

Dans cette recherche, nous nous sommes intéressés à la place tenue par l'expérience des valeurs dans le développement professionnel d'étudiants en situation d'alternance. Aborder la dimension axiologique sous cet angle nous a permis de faire émerger de nouvelles questions. La première étude menée auprès des référents à conduit à mettre en évidence l'usage évaluatif que ceux-ci font du positionnement professionnel des stagiaires : le regard sur les valeurs perçues revêt tantôt un caractère normatif dans une visée de contrôle, tantôt un caractère plus compréhensif dans une visée de reconnaissance. La deuxième étude, menée auprès des étudiants, met en évidence deux figures axiologiques de la professionnalité au travers desquelles s'organisent progressivement les modes de résolution des conflits de valeurs éprouvés dans le travail.

Bien que la méthodologie de recherche choisie exclue toute approche comparative, nous pouvons toutefois pointer un certain nombre d'éléments qui émergent de la mise en relation de ces deux recherches. Le point de vue des référents diverge ici de celui des stagiaires sur le rôle qu'occupent les valeurs dans le développement professionnel. Les référents, s'appuyant sur un ensemble de valeurs déclarées qui participent de leur ethos professionnel, considèrent le positionnement professionnel des stagiaires sous l'angle de l'acculturation ou de façon moins dynamique sous celui de la congruence. Il s'agit pour eux d'évaluer la proximité des valeurs signifiées avec celles de l'organisation. Les valeurs personnelles sont valorisées, dès lors qu'elles se rapprochent de celles qui ont cours et 
prennent dans le discours de certains référents la forme de pré-requis. Sont absents des propos les risques connus de simulation de rôle (Davis, op. cit.) ou bien la dimension professionnalisante du conflit de valeurs. Du côté des étudiants, l'harmonie axiologique relève d'un idéal vite dépassé : rares sont ceux qui échappent à l'expérience de l'incertitude, du désaccord, ou du conflit, fût-il intérieur. Loin de déplorer cet état de fait, les écrits témoignent du lien fait avec la perspective de développement : "Cette remise en question du sujet, de ses valeurs est difficile car d'autres références apparaissent mais c'est un passage obligatoire pour pouvoir les intégrer et construire son éthique professionnelle (5)». Se professionnaliser, c'est probablement répondre à la question du rapport entre valeurs individuelles et normes collectives, entre contraintes de l'action et de l'environnement et responsabilité des acteurs, entre identité héritée et identité professionnelle.

Nous conclurons ce texte en évoquant les quelques limites, questions ou perspectives qui peuvent être dégagées à l'issue de cette recherche :

- Notre exploration de la participation des valeurs et des conflits de valeurs aux processus de transformation opérant en cours de professionnalisation, nous conduit à mettre en travail l'hypothèse suivante : la dimension axiologique est un caractère spécifique du développement professionnel s'ajoutant à ceux existant (opératoire, cognitif, affectif et identitaire).

- Pour ne pas faire de cette dimension axiologique un élément limité aux effets contextuels de la formation et de l'insertion, mais bien un élément de professionnalisation et de professionnalité, il importerait de développer une réelle approche comparative des points de vue des étudiants et des professionnels. Dans cette optique, quelques considérations méthodologiques s'imposent : homogénéisation des modes de recueil de données et des méthodes d'analyse, nécessité d'approches longitudinales.

- Notre recherche pourrait laisser croire que la figure du professionnel responsable, assumant la part subjective de la délibération, serait l'apanage des étudiant en formation. Le primat de la normativité irait-il de soi parmi les collectifs expérimentés? Il serait intéressant d'étudier la place que les référents, interrogés cette fois-ci au regard de leur propre expérience et non de leur statut de tuteur, font aux conflits de valeurs dans leur pratique.

- Les divergences de conception entre référents et étudiants, qui devront être confirmées, appellent la remarque suivante. Elles peuvent n'être qu'un effet des rôles énonciatifs assumés par les acteurs. Le référent se positionnerait alors davantage comme un éventuel recruteur que comme un accompagnateur du développement, face à l'instance universitaire que représente son interlocuteur, instance à laquelle il laisse peut-être le rôle d'accompagnement.

- Les représentations associées au développement professionnel mériteraient d'être mieux documentées auprès des étudiants, des formateurs et des professionnels : en effet les éléments de réflexion apportés concernant l'expérience axiologique nous amènent à questionner l'allant de soi positif du changement en formation. 


\section{Bibliographie}

Ardoino, J. (1989). De la clinique. Réseaux, 55-57.

Aubert, N., Gaulejac, V. (1991). Le coût de l'excellence. Paris : Seuil.

Bachelard, G. (1938). La formation de l'esprit scientifique. Paris : Vrin.

Barbier, J.-M. (2003). Préférences d'engagement, représentations finalisantes et valeurs signifiées. In J.-M. Barbier (Ed.), Valeurs et activités professionnelles, Séminaire du Centre de Recherche sur la Formation du Cnam. Paris : L'Harmattan, 115-166.

Bardin, L. (2003). L'analyse de contenu. Paris : Presses Universitaires de France.

Bateson, G. (1971). La Cérémonie du Naven. Paris : Minuit.

Beckers, J. (2007). Compétences et identité professionnelles. L'enseignement et autres métiers de l'interaction humaine. Bruxelles : De Boeck.

Bedin, V. (2007). Faire le choix du concept d' « évaluation-conseil » en éducation et formation. Les Dossiers des Sciences de l'Éducation, ${ }^{\circ} 18,131-140$.

Bedin, V. (2008). Comment des professionnels reconnaissent une formation universitaire ? In A. Jorro (Ed.), La reconnaissance professionnelle en éducation. Evaluer, valoriser, légitimer. Ottawa : Presses Universitaires d'Ottawa, 77-99.

Boudon, R. (1999). Le sens des valeurs. Paris : Presses universitaires de France.

Bourdieu, P. (1980). Le sens pratique. Paris : Minuit.

Bourgeois, E. (2006). Tensions identitaires et engagement en formation. In J.-M. Barbier, E. Bourgeois, G. de Villers, M. Kaddouri (Eds.), Constructions identitaires et mobilisation des sujets en formation. Paris : L'Harmattan, 65-120.

Braem, S., Aballéa, F. (2002). La notion de professionnalité : un concept «transfert». Recherche sociale, 163, 4-15.

Cifali, M. (1996). Démarche clinique, formation et écriture. In L. Paquay, M. Altet, E. Charlier, P. Perrenoud (Eds.), Former des enseignants professionnels. Quelles stratégies? Quelles compétences? Bruxelles : De Broeck : 119-135.

Courpasson, D., Dany, F. (2008). Les cadres rebelles. Les Grands Dossiers des Sciences Humaines, $\mathrm{n}^{\circ} 12,72-75$.

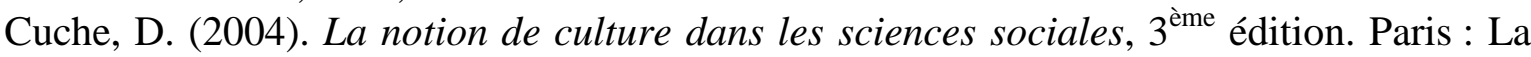
Découverte.

Davis, F. (1968). Professional Socialization as Subjective Experience : the Process of Doctrinal Conversion amoung Student Nurses. In H. Becker, B. Geer, D. Riesman et R. Weiss (Eds.), Institution and the Person : Essays in Honor of Everett Hughes. Chicago : Free Press, 235-251.

De Certeau, M. (1981). L'invention du quotidien 1. L'art de faire. Paris : Seuil.

Dubar, C. (1994). Identités collectives et individuelles dans le champ professionnel. In M. De Coster \& F. Pichault (Ed.), Traité de sociologie du travail, Bruxelles : De Boeck, 363-379.

Dubar, C. (2006). La socialisation. Construction des identités sociales et professionnelles. Paris : Armand Colin.

Dubet, F. (2002). Le déclin de l'institution. Paris : Seuil.

Durand, G. (1999). Introduction générale à la bioéthique. Histoire, concepts et outils. Montréal : Cerf, Fides.

Ferry, J.-M. (2002). Valeurs et normes. La question de l'éthique. Bruxelles : Editions de l'Université de Bruxelles.

Fournet, M. (2007). L'évaluation du processus d'auto-formation des cadres européens de la net-économie. In A. Jorro (Ed.), Evaluation et développement professionnel. Paris : L'Harmattan, 35-53. 
Jorro, A. (1999). L'instance évaluative dans les pratiques professionnelles des enseignants. Questions de recherche en Education, 1, 251-257.

Jorro, A. (2005). Le processus de professionnalisation. 9èmes Journées Nationales d'Etudes des Cadres de Santé, Institut de Formation des Cadres de Santé - CHU de Montpellier. Montpellier, 20 et 21 janvier 2005.

Jorro, A. (dir.), (2007). Evaluation et développement professionnel. Paris : L'Harmattan.

Kaddouri, M., Lespessailles, C., Maillebouis, M., Vasconcellos, M. (Eds.), (2008). La question identitaire dans le travail et la formation. Paris : L'Harmattan.

Lahire, B. (2001). L'homme pluriel : les ressorts de l'action. Paris : Nathan.

Legault, G. A. (1999). Professionnalisme et délibération éthique. Québec: Presse de l'Université du Québec.

Legault. G. (dir.), (2003). Crise d'identité professionnelle professionnalisme. Québec: Presse de l'Université du Québec.

Lecointe, M. (2007) L'évaluation : rationalités et imaginaires. In A. Jorro (Ed.), Evaluation et développement professionnel. Paris : L'Harmattan, 215-227.

Lhotellier, A. (2001). Tenir Conseil. Délibérer pour agir. Paris : Seli Arslan.

Massé, R. (2003). Ethique et santé publique. Enjeux, valeurs et normativité. Québec: Presses de l'Université Laval.

Osty, F. (2003). Le désir de métier. Engagement, identité et reconnaissance au travail. Rennes : Presses universitaires de Rennes.

Osty, F. (2008). L'identité au travail à l'épreuve de la crise. In M. Kaddouri, C. Lespessailles, M. Maillebouis, M. Vasconcellos (Eds.), (2008). La question identitaire dans le travail et la formation. Paris : L'Harmattan, 69-81.

Sainsaulieu, R. (1988). L'identité au travail. Les effets culturels de l'organisation. Paris : Presses de la fondation nationale des sciences politiques.

Tardif, M., Lessard, C. (2004). Le travail enseignant au quotidien. Contribution à l'étude du travail dans les métiers et les professions d'interactions humaines. Laval : Presses universitaires de Laval.

Wittorski, R. (2007). Professionnalisation et développement professionnel. Paris : L'Harmattan. 